NOTA CIENTÍFICA

\title{
Surrogacía taxonómica en bosque montano andino
}

\author{
Taxonomic surrogacy in andean montane forests
}

\author{
Salvador Herrando Pérez ${ }^{1,2}$ y María de los Ángeles La Torre-Cuadros ${ }^{3,4^{*}}$
}

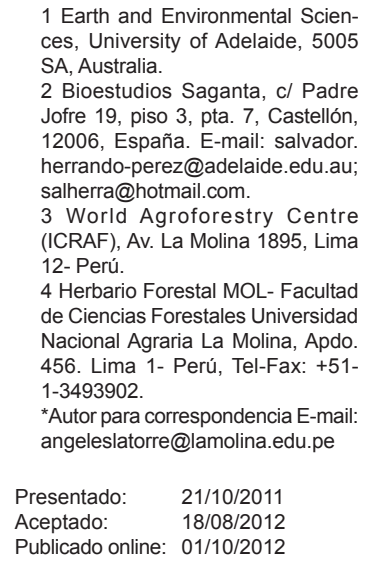

\begin{abstract}
Resumen
En ecología, los surrogados son variables ambientales o bióticas que representan la biodiversidad de un área Su uso permite afrontar limitaciones logísticas y/o desconocimiento taxonómico para muestrear e identificar especies, y se ha convertido en una herramienta importante en conservación y gestión. En este trabajo, ilustramos un método de surrogacía basado en ordenaciones multivariadas, y lo aplicamos a datos de cobertura arbórea en el bosque montano de Chanchamayo (Andes, Perú).
\end{abstract}

Palabras clave: Chanchamayo, Conservación, Escalamiento multi-dimensional, Ensamblajes de árboles, Perú

\section{Abstract}

Ecological surrogates are environmental or biotic proxies for biodiversity quantification. Their application circumvents logistic constraints and taxonomic voids to sample and identify species, and has become an important tool in conservation and management. In this study, we illustrate a surrogate method using multivariate ordinations, and we apply it to tree data from the Chanchamayo montane forest (Andes, Peru).

Keywords: Chanchamayo, Conservation, Multi-dimensional scaling, Peru, Tree assemblages

\section{Introducción}

El concepto de surrogados fue introducido en la biología de la conservación en la segunda mitad del siglo pasado, y definido como los grupos taxonómicos bien conocidos, cuyo monitoreo es relativamente sencillo y cuya diversidad representa la diversidad de otros grupos taxonómicos. La gestión de las aéreas protegidas pueden verse afectadas por las dificultades surgidas ante el enorme esfuerzo que implica muestrear grandes extensiones de hábitat y la incertidumbre taxonómica (y por supuesto genética) de muchos componentes de la biota; es por ello que la utilización de surrogados se ha convertido en herramienta imprescindible en estrategias modernas de selección y gestión de reservas (Margules $\&$ Pressey 2000, Pressey 2004, Sarkar et al. 2006), incluso a nivel global (Balmford et al. 1996, Myers et al. 2000, Hughes et al. 2002, Lamoreux et al. 2006).

Con su creciente aplicación, el concepto de surrogado se ha expandido en varias direcciones, requiriendo definición de conceptos afines como 'complementariedad', 'congruencia', 'irremplazabilidad' o 'vulnerabilidad' (Sarkar et al. 2006). Se han descrito distintos tipos de surrogados (especies 'indicadoras', 'paraguas', 'bandera', 'focales', entre otras) que atienden objetivos de conservación particulares (Lambeck 1997, Caro \& O'Doherty 1999, Favreau et al. 2006, Rodrigues \& Brooks 2007), y también métodos que analizan surrogados de riqueza tanto de especies (e.g. Price et al. 2006, Lewandowski et al. 2010, Mazaris et al. 2010, van Weerd \& de Haes 2010) como de ensamblajes de especies (e.g. Oliver et al. 1998, Pharo et al. 1999, Hooper et al. 2002, Chiarucci et al. 2005, Arponen et al. 2008, Cushman et al. 2010). Por otra parte, un surrogado ya no es necesariamente una planta o un animal, puede ser también una variable ambiental (Faith \& Walker 1996, Wessels et al. 1999, Faith 2003, Lassau $\&$ Hochuli 2008, Howell 2010), y esos surrogados ambientales son de innegable utilidad en ecosistemas de alta diversidad en hábitats de grandes proporciones como arrecifes coralinos (Mumby et al. 2008) o selvas tropicales (Costa et al. 2005). Fi- nalmente, la surrogacía también ha abordado si diversidad a nivel de especie puede ser capturada con identificaciones taxonómicas de individuos a nivel de género hasta orden (e.g. Grelle 2002, Olsgard et al. 2003, Cardoso et al. 2004, Villasenor et al. 2005, Li et al. 2006, Mandelik et al. 2007), morfoespecies (Oliver \& Beattie 1996, Ramsay et al. 2006, Abadie et al. 2008), gremios funcionales (Gitay et al. 1999, Bady et al. 2005, Mayfield et al. 2005, Cabeza et al. 2008), y biota muerta (Warwick \& Light 2002). En todos esos casos, los surrogados taxonómicos tratan de eludir los costos logísticos de una identificación sistemática precisa. Esencialmente se trata de cuantificar biodiversidad, con menos esfuerzo y menos dinero pero manteniendo la fiabilidad de la estimación, y por lo tanto siempre conlleva un compromiso entre costos y fiabilidad.

Este trabajo examina esquemáticamente parte del análisis de surrogacía taxonómica en bosque montano andino del Valle de Chanchamayo (Junín, Perú) realizado por La Torre-Cuadros et al. (2007), de manera que pueda ser replicable para investigadores ajenos a este tipo de técnicas. En concreto, el análisis determina si la diferenciación de ensamblajes arbóreos en varias parcelas florísticas coincide para 6 niveles taxonómicos (morfoespecie, especie, género, familia, orden, phylum). A falta de robustecer la fiabilidad de nuestras conclusiones con un número mayor de parcelas, los resultados sugieren que, para el bosque montano de Chanchamayo, la presencia/ausencia de familias puede ser un surrogado taxonómico fiable del recambio de especies arbóreas.

\section{Datos florísticos}

Los datos biológicos son de abundancia relativa de árboles ( $>10 \mathrm{~cm}$ de diámetro a la altura del pecho) en 7 parcelas de 1 hectárea en el Valle de Chanchamayo. Las parcelas representan distintas alturas, pendientes y laderas de esta región de la Cordillera Andina (ver La Torre-Cuadros et al. 2007), incluyendo un sitio de estudio en el recientemente creado Santuario Nacional de Pampa Hermosa. Se construyeron 12 bases de datos rectangulares de abundancia relativa de taxa en las 7 parcelas (Fig. 1): 


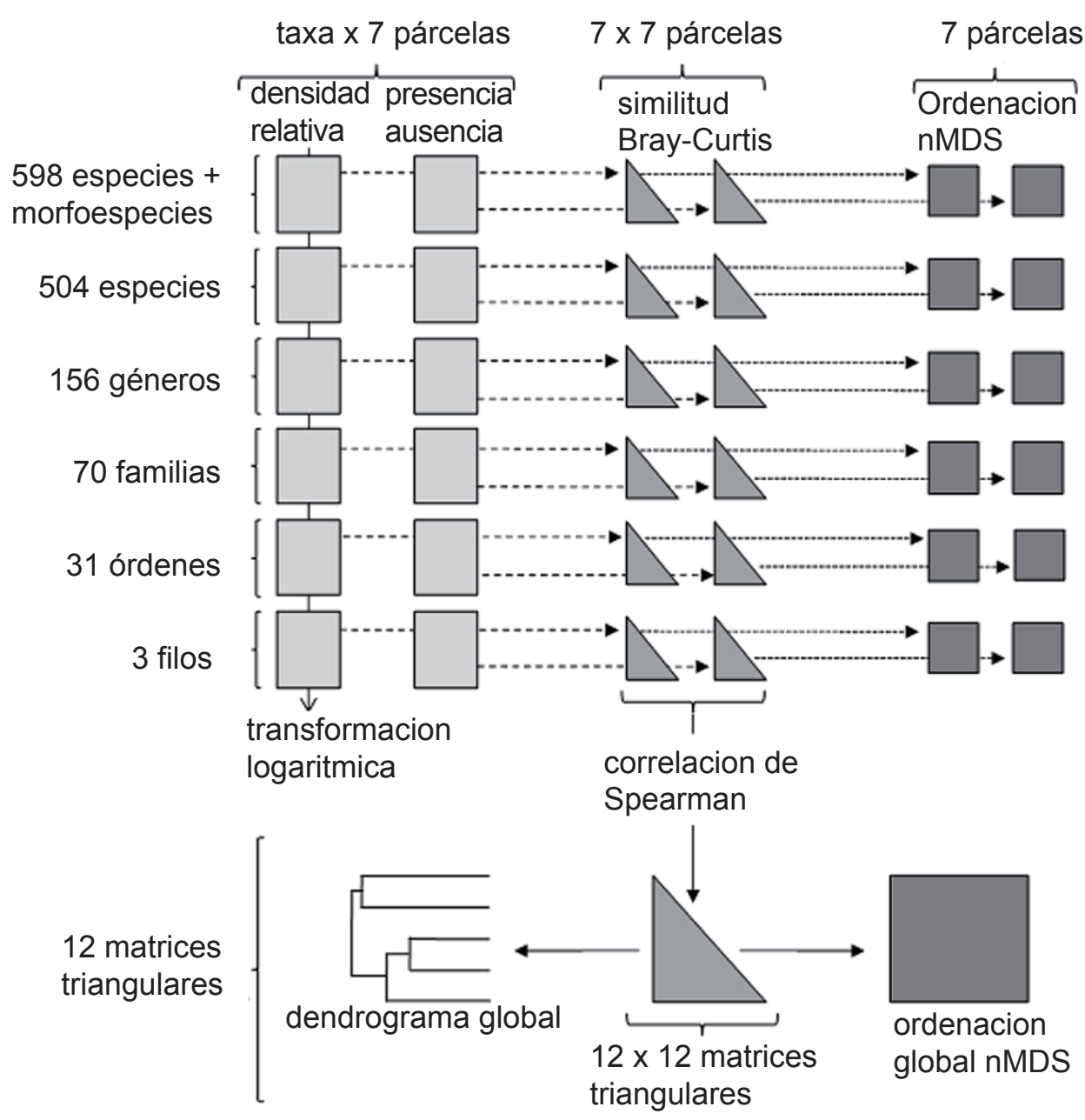

Figura 1. Esquema del análisis de surrogacía taxonómica del estrato arbóreo de 7 parcelas florísticas (1 hectárea) en el Valle de Chanchamayo. Se produce una ordenación nMDS global y un dendrograma global a partir de similitudes Bray-Curtis [matrices triangulares pequeñas] basadas en datos de abundancia relativa (presencia/ausencia, y densidades logarítmicas) para 6 niveles taxonómicos (con inclusión de morfoespecies en uno de los niveles) [matrices rectangulares]. Se utilizó correlación de Spearman entre las 12 matrices triangulares pequeñas para construir la matriz triangular grande empleada en la ordenación y clasificación globales.

6 de esas bases de datos incluyeron densidades transformadas logarítmicamente para cada taxón, y las otras 6 contenían presencia/ausencia (unos y ceros) de taxones. La transformación permite que las ordenaciones sean sensibles a la abundancia relativa de especies dominantes y especies de menor abundancia. Las especies fueron agrupadas por género/familia/orden/phylum, de manera que la densidad relativa en cada uno de esos niveles taxónomicos por parcela resultó de sumar las densidades relativas de todas las especies pertenecientes a cada nivel (Fig. 1). Un total de 94 taxones de árboles pudieron ser diferenciados morfológicamente sin asignación taxonómica (morfoespecies), de manera que también se construyeron dos matrices de densidad relativa: una con especies identificadas y otra con especies identificadas más morfoespecies. En el caso de presencia/ausencia, las matrices rectangulares incluyeron 'unos' para aquellas parcelas en las que los distintos niveles taxonómicos estaban representados por al menos una especie. Los ensamblajes de árboles fueron estudiados mediante dos métodos de estadística multivariada en el programa Primer (Clarke \& Gorley 2006). Por 'ensamblaje' de árboles se siguió la definición de Fauth et al. (1996) (i.e., grupos de especies emparentadas filogenéticamente que cohabitan en la misma comunidad), y diferencias en ensamblajes entre las 7 parcelas de estudio indicaron recambios en la composición de taxones (datos presencia/ausencia) más cambios en la densidad relativa de esos taxones (datos transformados logarítmicamente).

\section{Análisis de recambio de taxones entre parcelas}

La estructura de los ensamblajes en análisis individuales para cada nivel taxonómico se realizó mediante 'Escalamiento multidimensional no-métrico' (nMDS; Kruskal 1964). nMDS utiliza un procedimiento de maximización numérica para representar las parcelas de estudio en un gráfico de ordenación de acuerdo a una medida de similitud, generando una medida de fiabilidad denominada stress (stress $<0,1$ corresponde con ordenaciones muy fiables). En la ordenación, parcelas con ensamblajes arbóreos relativamente semejantes (menor recambio de taxones entre ellas) aparecerán más cercanas que parcelas con ensamblajes más discrepantes (mayor recambio de taxones). Para cuantificar el recambio de taxones entre parcelas, se utilizó similitud BrayCurtis por sus comprobadas propiedades frente a otros índices, particularmente la adición de muestras a un análisis (por ejemplo, tras una nueva campańa de muestreo en campo) no afecta los valores de similitud ya calculados entre muestras previas, la doble ausencia de un especie en dos localidades no contribuye ninguna similitud entre muestras, y la similitud es máxima o mínima cuando, respectivamente, dos muestras son idénticas o 


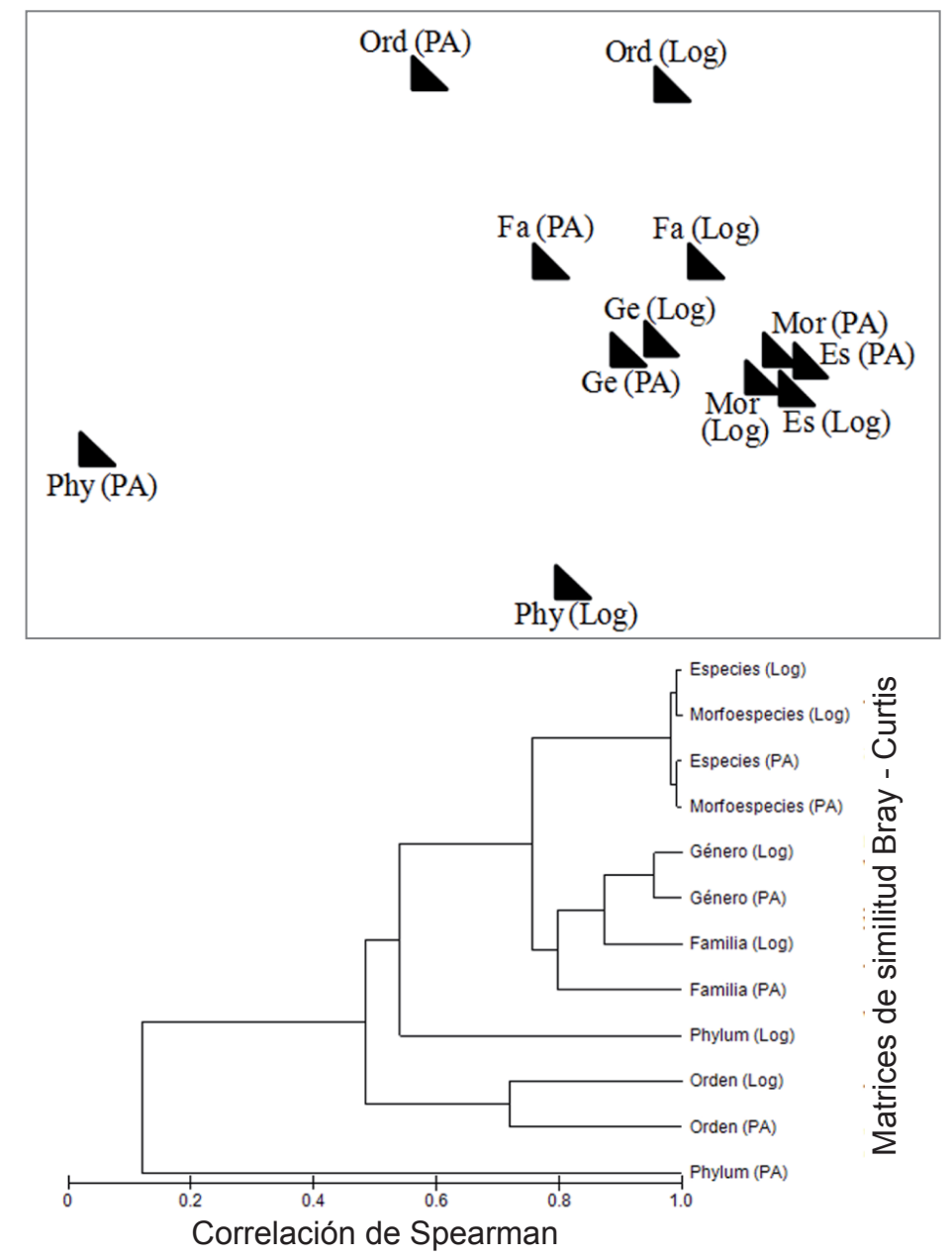

Figura 2. Ordenación nMDS global (arriba, Stress $=0.04)$ y Dendrograma global (abajo) del análisis de surrogacía taxonómica del estrato arbóreo de 7 parcelas florísticas (1 hectárea) en el Valle de Chanchamayo, para 6 niveles taxonómicos y similitudes Bray-Curtis entre parcelas. Identificaciones a nivel de morfoespecie, especie, género y familia, tanto con presencia/ausencia de taxones (PA) como con sus densidades transformadas con logaritmos (Log), revelan relativamente altas correlaciones de Spearman ( $>0.7)$. Las correlaciones a nivel de orden y phylum son de menor magnitud.

no comparten ninguna especie (ver Clarke et al. 2006). Se calculó un valor de similitud Bray-Curtis entre cada pareja posible de parcelas $\mathrm{y}$, como resultado, cada matriz rectangular 'taxones $\mathrm{x}$ 7 parcelas' se convirtió en una matriz triangular ' 7 x 7 parcelas' compuesta por similitudes Bray-Curtis. Los nMDs están basados en esas matrices de similitud. El resultado de esta parte del análisis son 12 ordenaciones nMDS en base a otras tantas matrices triangulares construidas a partir de datos de densidad o presencia/ausencia para los 6 niveles taxonómicos (Fig. 1).

Los nMDS para morfoespecies, especies, géneros y familias pueden ser consultadas en La Torre Cuadros et al. (2007). A nivel de especie (con y sin morfoespecies) se distinguieron 3 tipos de ensamblajes: 1 parcela de bosque montano bajo (Pampa Hermosa), y 6 de bosque montano propiamente con ensamblajes distintos en la franja occidental (Pichita, 2 parcelas) y en la franja oriental (Génova y San Ramón, 4 parcelas) de esta parte de la Cordillera Andina.

\section{Análisis de surrogados taxonómicos}

La Ordenación de segundo orden (SSO) (Somerfield \& Clarke, 1995) permite evaluar el grado de parecido de las 12 ordenaciones nMDS producidas en el apartado anterior. Esa semejanza se cuantifica con una simple correlación (normalmente
Spearman o Kendall) entre cada dúo de matrices triangulares (Mantel 1967). Como cualquier índice de correlación, aquí también se relacionan medidas pareadas de dos variables: en este caso similitudes Bray-Curtis entre matrices triangulares. Disponemos de 12 matrices triangulares de $7 \times 7$ parcelas, y por ello el SSO calcula un valor de correlación entre cada dúo de matrices triangulares (Fig. 1). El resultado es una matriz triangular global de tamaño 12 x 12 y rellena de correlaciones que, tomadas como índices de similitud, sirven para producir una ordenación nMDS global. Los puntos en esa ordenación global representan cada matriz triangular. Aquellas matrices triangulares que producen ordenaciones parecidas de parcelas (correlaciones altas implican un patrón parecido de recambio de taxones) aparecerán más cercanas entre sí en el nMDS global que aquellas matrices triangulares que producen ordenaciones contrastantes (correlaciones bajas). El nMDS global fue complementado con un dendrograma sobre la matriz triangular global utilizando promediado grupal como criterio de agrupamiento (Day \& Edelsbrunner 1984).

Los resultados de este análisis indican correlaciones bastantes altas $(>0,7)$ en el recambio de taxones entre parcelas a nivel de especie (con y sin morfoespecies), género y familia, tanto cuando se utiliza presencia/ausencia como cuando se usan densidades 
logarítmicas (Fig. 2). Las correlaciones disminuyen ostensiblemente cuando se compara el recambio de especies con el recambio de órdenes o phyla entre parcelas (Fig. 2). La Torre Cuadros et al. (2007) describen la semejanza de las ordenaciones a nivel de especie, género y familia con datos de presencia/ausencia, abundancias relativas y áreas basales (crudas y logarítmicas), incluyendo además morfogéneros y morfofamilias.

\section{Comentario final}

La franja tropical de la Cordillera de los Andes constituye uno de los 25 principales "puntos calientes" de biodiversidad global, es decir alto endemismo coincide geográficamente con alta tasa de degradación ambiental (Myers et al. 2000). Su gran extensión ( $-300,000 \mathrm{~km} 2,25 \%$ protegidos) explica que lidere los registros globales de plantas y vertebrados endémicos, y hace de la aplicación de surrogados una necesidad para manejar su conservación. Este estudio sugiere que la diversidad de ensamblajes arbóreos del bosque montano del Valle de Chanchamayo podría ser monitoreada óptimamente estimando presencia/ ausencia de familias en parcelas de 1 hectárea y obviando taxones poco conocidos.

Es importante señalar que estos resultados son provisionales y deberán confrontarse con datos arbóreos para un mayor número de parcelas en futuros estudios. Sería interesante ubicar nuevas parcelas a lo largo de las Yungas peruanas para direccionar las ya emergentes y otras nuevas investigaciones, y añadir nuevos objetivos de estudio, en particular la utilidad del surrogado arbóreo en la identificación de distintos grados de perturbación antropogénica y como indicador de diversidad de grupos zoológicos. La iniciativa puede tener amplio impacto en instituciones patrocinadoras de conservación internacional y en los grandes proyectos de infraestructura y extracción.

Muchos de los estimadores de diversidad usados en los análisis de surrogacía, sean de diversidad de especies o ensamblajes, y en particular cuando el tamaño muestral es pequeño, necesitarán de procedimientos como jackknifing o bootstrapping que permitan calcular intervalos de confianza (e.g. Hellmann \& Fowler 1999, Anderson \& Ashe 2000), o randomización para controlar la presencia azarosa de especies (e.g. Warwick \& Light 2002, Lamoreux et al. 2006, Cushman et al. 2010). Los métodos de ordenación multivariada aquí utilizados son conceptualmente sencillos, y con mínimas asunciones matemáticas. Referencia a la gama disponible de paquetes estadísticos y bibliografía sobre métodos de ordenación puede consultarse en red 'ordination. okstate.edu' y en Herrando-Pérez et al. (2008), y especial mención merecen los paquetes 'Vegan' y 'Ade4' ejecutables gratuitamente en lenguaje de programación R (R:DevelopmentCore-Team 2010).

En último término queremos resaltar que los surrogados no eliminan la necesidad de estudiar especies y el papel imprescindible que el taxónomo especializado cumple en el inventariado de la biodiversidad. El incremento de especies identificadas subrayará el valor de cualquier área protegida, todavía más si conduce a la descripción de taxones nuevos para la ciencia e identificación de endemismos de gran utilidad en biogeografía, macroecología, conservación y bioprospección.

\section{Agradecimientos}

Al financiamiento otorgado a M.A. La Torre-Cuadros por la beca semilla de Iniciativa de Liderazgo para la Conservación de la Amazonía (ACLI) de la Universidad de Florida apoyada por la Iniciativa Andes-Amazónica de la Fundación Gordon y Betty Moore y al Dr. Kenneth Feeley por sus valiosas sugerencias al manuscrito.

\section{Literatura citada}

Abadie, J. C., Andrade, C., Machon, N. \& Porcher, E. 2008. On the use of parataxonomy in biodiversity monitoring: a case study on wild flora. Biodiversity and Conservation, 17, 3485-3500.

Anderson, R. S. \& Ashe, J. S. 2000. Leaf litter inhabiting beetles as surrogates for establishing priorities for conservation of selected tropical montane cloud forests in Honduras, Central America (Coleoptera; Staphylinidae, Curculionidae). Biodiversity and Conservation, 9, 617-653.

Arponen, A., Moilanen, A. \& Ferrier, S. 2008. A successful community-level strategy for conservation prioritization. Journal of Applied Ecology, 45, 1436-1445.

Bady, P., Doledec, S., Fesl, C., Gayraud, S., Bacchi, M. \& Scholl, F. 2005. Use of invertebrate traits for the biomonitoring of European large rivers: the effects of sampling effort on genus richness and functional diversity. Freshwater Biology, 50, 159-173.

Balmford, A., Green, M. J. B. y Murray \& M. G. 1996. Using higher-taxon richness as a surrogate for species richness .1. Regional tests. Proceedings of the Royal Society of London Series B-Biological Sciences, 263, 1267-1274.

Cabeza, M., Arponen, A. \& Van Teeffelen, A. 2008. Top predators: hot or not? A call for systematic assessment of biodiversity surrogates. Journal of Applied Ecology, 45, 976-980.

Cardoso, P., Silva, I., de Oliveira, N. G. \& Serrano, A. R. M. 2004. Higher taxa surrogates of spider (Araneae) diversity and their efficiency in conservation. Biological Conservation, 117, 453-459.

Caro, T. M. \& O'Doherty, G. 1999. On the use of surrogate species in conservation biology. Conservation Biology, 13, 805-814.

Chiarucci, A., D'Auria, F., De Dominicis, V., Lagana, A., Perini, C. \& Salerni, E. 2005. Using vascular plants as a surrogate taxon to maximize fungal species richness in reserve design. Conservation Biology, 19, 1644-1652.

Clarke, KR, Gorley, RN, 2006. PRIMER v6: User Manual/Tutorial. PRIMER-E, Plymouth.

Clarke, K. R., Somerfield, P. J. \& Chapman, M. G. 2006. On resemblance measures for ecological studies, including taxonomic dissimilarities and a zero-adjusted Bray-Curtis coefficient for denuded assemblages. Journal of Experimental Marine Biology and Ecology, 330, 55-80.

Costa, F. R. C., Magnusson, W. E. \& Luizao, R. C. 2005. Mesoscale distribution patterns of Amazonian understorey herbs in relation to topography, soil and watersheds. Journal of Ecology, 93, 863-878.

Cushman, S. A., McKelvey, K. S., Noon, B. R. \& McGarigal, K. 2010. Use of Abundance of One Species as a Surrogate for Abundance of Others. Conservation Biology, 24, 830-840.

Day, W. H. E. \& Edelsbrunner, H. E. 1984. Efficient algorithms for agglomerative hierarchical clustering methods Journal of Classification, 1, 7-24.

Faith, D. P. 2003. Environmental diversity (ED) as surrogate information for species-level biodiversity. Ecography, 26, 374-379.

Faith, D. P. \& Walker, P. A. 1996. Environmental diversity: On the best-possible use of surrogate data for assessing the relative biodiversity of sets of areas. Biodiversity and Conservation, 5, 399-415.

Fauth, J. E., Bernardo, J., Camara, M., Resetarits, W. J., VanBuskirk, J. \& McCollum, S. A. 1996. Simplifying the jargon of community ecology: A conceptual approach. The American Naturalist, 147, 282-286. 
Favreau, J. M., Drew, C. A., Hess, G. R., Rubino, M. J., Koch, F. H. \& Eschelbach, K. A. 2006. Recommendations for assessing the effectiveness of surrogate species approaches. Biodiversity and Conservation, 15, 3949-3969.

Gitay, H., Noble, I. R. \& Connell, J. H. 1999. Deriving functional types for rain-forest trees. Journal of Vegetation Science, 10, 641-650.

Grelle, C. E. V. 2002. Is higher-taxon analysis an useful surrogate of species richness in studies of Neotropical mammal diversity? Biological Conservation, 108, 101-106.

Hellmann, J. J. \& Fowler, G. W. 1999. Bias, precision, and accuracy of four measures of species richness. Ecological Applications, 9, 824-834.

Herrando-Pérez, S., Baratti, M. \& Messana, G. 2008. Subterranean ecological research and multivariate statistics: A review (1945-2006). Journal of Cave and Karst Studies, 70, 120-128.

Hooper, J. N. A., Kennedy, J. A. \& Quinn, R. J. 2002. Biodiversity 'hotspots', patterns of richness and endemism, and taxonomic affinities of tropical Australian sponges (Porifera). Biodiversity and Conservation, 11, 851-885.

Howell, K. L. 2010. A benthic classification system to aid in the implementation of marine protected area networks in the deep/ high seas of the NE Atlantic. Biological Conservation, 143, 1041-1056.

Hughes, T. P., Bellwood, D. R. \& Connolly, S. R. 2002. Biodiversity hotspots, centres of endemicity, and the conservation of coral reefs. Ecology Letters, 5, 775-784.

Kruskal, J. B. 1964. nonMetric Multidimensional scaling - a numerical method. Psychometrika, 29, 115-129.

La Torre-Cuadros, M., Herrando-Pérez, S. \& Young, K. R. 2007. Diversity and structural patterns for tropical montane and premontane forests of central Peru, with an assessment of the use of higher-taxon surrogacy. Biodiversity and Conservation, 16, 2965-2988.

Lambeck, R. J. 1997. Focal species: A multi-species umbrella for nature conservation. Conservation Biology, 11, 849-856.

Lamoreux, J. F., Morrison, J. C., Ricketts, T. H., Olson, D. M., Dinerstein, E., McKnight, M. W. \& Shugart, H. H. 2006. Global tests of biodiversity concordance and the importance of endemism. Nature, 440, 212-214.

Lassau, S. A. \& Hochuli, D. F. 2008. Testing predictions of beetle community patterns derived empirically using remote sensing. Diversity and Distributions, 14, 138-147.

Lewandowski, A. S., Noss, R. F. \& Parsons, D. R. 2010. The effectiveness of surrogate taxa for the representation of biodiversity. Conservation Biology, 24, 1367-1377.

Li, Z. Q., Yu, D., Xiong, W., Wang, Q. \& Tu, M. H. 2006. Testing the higher-taxon approach: a case study of aquatic marcophytes in China's northwest arid zone and its implications for conservation. Biodiversity and Conservation, 15, 3401-3416.

Mandelik, Y., Dayan, T., Chikatunov, V. \& Kravchenko, V. 2007. Reliability of a higher-taxon approach to richness, rarity, and composition assessments at the local scale. Conservation Biology, 21, 1506-1515.

Mantel, N. 1967. The detection of disease clustering and a generalized regression approach. Cancer Research, 27, 209-220.

Margules, C. R. \& Pressey, R. L. 2000. Systematic conservation planning. Nature, 405, 243-253.

Mayfield, M. M., Boni, M. E., Daily, G. C.\& Ackerly, D. 2005. Species and functional diversity of native and humandominated plant communities. Ecology, 86, 2365-2372.
Mazaris, A. D., Kallimanis, A. S., Tzanopoulos, J., Sgardelis, S. P. \& Pantis, J. D. 2010. Can we predict the number of plant species from the richness of a few common genera, families or orders? Journal of Applied Ecology, 47, 662-670.

Mumby, P. J., Broad, K., Brumbaugh, D. R., Dahlgren, C. P., Harborne, A. R., Hastings, A., Holmes, K. E., Kappel, C. V., Micheli, F. \& Sanchirico, J. N. 2008. Coral reef habitats as surrogates of species, ecological functions, and ecosystem services. Conservation Biology, 22, 941-951.

Myers, N., Mittermeier, R. A., Mittermeier, C. G., da Fonseca, G. A. B. \& Kent, J. 2000. Biodiversity hotspots for conservation priorities. Nature, 403, 853-858.

Oliver, I. \& Beattie, A. J. 1996. Designing a cost-effective invertebrate survey: A test of methods for rapid assessment of biodiversity. Ecological Applications, 6, 594-607.

Oliver, I., Beattie, A. J. \& York, A. 1998. Spatial fidelity of plant, vertebrate, and invertebrate assemblages in multiple-use forest in eastern Australia. Conservation Biology, 12, 822-835.

Olsgard, F., Brattegard, T. \& Holthe, T. 2003. Polychaetes as surrogates for marine biodiversity: lower taxonomic resolution and indicator groups. Biodiversity and Conservation, 12, 1033-1049.

Pharo, E. J., Beattie, A. J. \& Binns, D. 1999. Vascular plant diversity as a surrogate for bryophyte and lichen diversity. Conservation Biology, 13, 282-292.

Pressey, R. L. 2004. Conservation planning and biodiversity: Assembling the best data for the job. Conservation Biology, $18,1677-1681$.

Price, A. R. G., Vincent, L. P. A., Venkatachalam, A. J., Bolton, J. J. \& Basson, P. W. 2006. Concordance between different measures of biodiversity in Indian Ocean macroalgae. Marine Ecology-Progress Series, 319, 85-91.

R:Development-Core-Team 2010. Foundation for Statistical Computing, Vienna, Austria.

Ramsay, P. M., Kent, M., Reid, C. L. \& Duckworth, J. C. 2006. Taxonomic, morphological and structural surrogates for the rapid assessment of vegetation. Journal of Vegetation Science, 17, 747-754.

Rodrigues, A. S. L. \& Brooks, T. M. 2007. Shortcuts for biodiversity conservation planning: The effectiveness of surrogates. Annual Review of Ecology Evolution and Systematics, 38, 713-737.

Sarkar, S., Pressey, R. L., Faith, D. P., Margules, C. R., Fuller, T., Stoms, D. M., Moffett, A., Wilson, K. A., Williams, K. J., Williams, P. H. \& Andelman, S. 2006. Biodiversity conservation planning tools: Present status and challenges for the future. Annual Review of Environment and Resources, 31, 123-159.

Somerfield, P. J. \& Clarke, K. R. 1995. Taxonomic levels, in marine community studies, revisited. Marine Ecology-Progress Series, 127, 113-119.

van Weerd, M. \& de Haes, H. A. U. 2010. Cross-taxon congruence in tree, bird and bat species distributions at a moderate spatial scale across four tropical forest types in the Philippines. Biodiversity and Conservation, 19, 3393-3411.

Villasenor, J. L., Ibarra-Manriquez, G., Meave, J. A. \& Ortiz, E. 2005. Higher taxa as surrogates of plant biodiversity in a megadiverse country. Conservation Biology, 19, 232-238.

Warwick, R. M. \& Light, J. 2002. Death assemblages of molluscs on St Martin's Flats, Isles of Scilly: a surrogate for regional biodiversity? Biodiversity and Conservation, 11, 99-112.

Wessels, K. J., Freitag, S. \& van Jaarsveld, A. S. 1999. The use of land facets as biodiversity surrogates during reserve selection at a local scale. Biological Conservation, 89, 21-38. 
